\title{
Natural history of prostate cancer on active surveillance: stratification by MRI using the PRECISE recommendations in a UK cohort
}

\author{
Francesco Giganti ${ }^{1,2}$ (1) $\cdot$ Armando Stabile $^{2,3} \cdot$ Vasilis Stavrinides $^{2,4} \cdot$ Elizabeth Osinibi $^{2} \cdot$ Adam Retter $^{1,2}$. \\ Clément Orczyk $^{2,4}$ - Valeria Panebianco ${ }^{5}$ - Bruce J. Trock ${ }^{6}$ - Alex Freeman ${ }^{7}$ - Aiman Haider ${ }^{7}$. Shonit Punwani ${ }^{1,8}$. \\ Clare Allen $^{1} \cdot$ Alex Kirkham $^{1} \cdot$ Mark Emberton $^{2,4} \cdot$ Caroline M. Moore $^{2,4}$
}

Received: 1 July 2020 / Revised: 28 July 2020 / Accepted: 1 September 2020 / Published online: 30 September 2020

(C) The Author(s) 2020

\begin{abstract}
Objectives The PRECISE recommendations for magnetic resonance imaging (MRI) in patients on active surveillance (AS) for prostate cancer $(\mathrm{PCa})$ include repeated measurement of each lesion, and attribution of a PRECISE radiological progression score for the likelihood of clinically significant change over time. We aimed to compare the PRECISE score with clinical progression in patients who are managed using an MRI-led AS protocol.

Methods A total of 553 patients on AS for low- and intermediate-risk PCa (up to Gleason score $3+4$ ) who had two or more MRI scans performed between December 2005 and January 2020 were included. Overall, 2161 scans were retrospectively re-reported by a dedicated radiologist to give a PI-RADS v2 score for each scan and assess the PRECISE score for each follow-up scan. Clinical progression was defined by histological progression to $\geq$ Gleason score $4+3$ (Gleason Grade Group 3) and/or initiation of active treatment. Progression-free survival was assessed using Kaplan-Meier curves and log-rank test was used to assess differences between curves.

Results Overall, 165/553 (30\%) patients experienced the primary outcome of clinical progression (median follow-up, 74.5 months; interquartile ranges, 53-98). Of all patients, 313/553 (57\%) did not show radiological progression on MRI (PRECISE 1-3), of which 296/313 (95\%) had also no clinical progression. Of the remaining 240/553 patients (43\%) with radiological progression on MRI (PRECISE 4-5), 146/240 (61\%) experienced clinical progression $(p<0.0001)$. Patients with radiological progression on MRI (PRECISE 4-5) showed a trend to an increase in PSA density.

Conclusions Patients without radiological progression on MRI (PRECISE 1-3) during AS had a very low likelihood of clinical progression and many could avoid routine re-biopsy.
\end{abstract}

Electronic supplementary material The online version of this article (https://doi.org/10.1007/s00330-020-07256-z) contains supplementary material, which is available to authorized users.

Francesco Giganti

f.giganti@ucl.ac.uk

1 Department of Radiology, University College London Hospital NHS Foundation Trust, London, UK

2 Division of Surgery \& Interventional Science, University College London, 3rd Floor, Charles Bell House, 43-45 Foley St.,

London W1W 7TS, UK

3 Department of Urology and Division of Experimental Oncology, Vita-Salute San Raffaele University, Milan, Italy
4 Department of Urology, University College London Hospital NHS Foundation Trust, London, UK

5 Department of Radiological Sciences, Oncology and Pathology, Sapienza University of Rome, Rome, Italy

6 The James Buchanan Brady Urological Institute and Department of Urology, Johns Hopkins University School of Medicine, Baltimore, MD, USA

7 Department of Pathology, University College London Hospital NHS Foundation Trust, London, UK

8 Centre for Medical Imaging, University College London, London, UK 
Key Points

- Patients without radiological progression on MRI (PRECISE 1-3) during AS had a very low likelihood of clinical progression and many could avoid routine re-biopsy.

- Clinical progression was almost always detectable in patients with radiological progression on MRI (PRECISE 4-5) during AS.

- Patients with radiological progression on MRI (PRECISE 4-5) during AS showed a trend to an increase in PSA density.

Keywords Urogenital neoplasms $\cdot$ Prostatic neoplasms $\cdot$ Magnetic resonance imaging $\cdot$ Biopsy

$\begin{array}{ll}\text { Abbreviations } \\ \text { AS } & \text { Active surveillance } \\ \text { DCE } & \text { Dynamic contrast enhanced } \\ \text { DWI } & \text { Diffusion-weighted imaging } \\ \text { GGG } & \text { Gleason Grade Group } \\ \text { MRI } & \text { Magnetic resonance imaging } \\ \text { NICE } & \text { National Institute for Health and Care Excellence } \\ \text { PCa } & \text { Prostate cancer } \\ \text { PI-RADS } & \text { Prostate Imaging Reporting and Data System } \\ \text { PRECISE } & \text { Prostate Cancer Radiological Estimation } \\ & \text { of Change in Sequential Evaluation } \\ \text { PSA } & \text { Prostate-specific antigen } \\ \text { T2-WI } & \text { T2-weighted imaging } \\ \text { TRUS } & \text { Transrectal ultrasound }\end{array}$

\section{Introduction}

Most policy groups around the world now recommend active surveillance (AS) as an appropriate option for patients with low-risk prostate cancer $(\mathrm{PCa})[1,2]$. However, the current position is less than satisfactory as most of the evidence base to support its use is from single centres, no standard AS protocol exists and we lack a method of reliably ascertaining when true progression (by either grade or volume) occurs.

Multi-parametric MRI (mpMRI) has provided us with an opportunity to move away from random prostate biopsies and address the challenge of discriminating true progression from mere re-classification [3]. Recent evidence suggests that prebiopsy MRI should be performed before confirmatory systematic transrectal ultrasound (TRUS)-guided biopsies during AS, together with MRI-targeted biopsies when indicated [4]. The ASIST trial initially showed no difference in the upgrade rate between patients having standard re-biopsy and those having MRI with 2 cores targeted to a lesion (upgrade to Gleason Grade Group 2 (GGG 2) was $21 \%$ vs $23 \%$ respectively, $p=0.9$ ), although there were marked differences between the study centres [5]. However, after 2 years of follow-up, the authors found that baseline mpMRI before confirmatory biopsy resulted in 50\% fewer failures of surveillance and less progression to higher-grade cancer, confirming the value of mpMRI during AS [6].
Addressing the serial use of MRI in AS, there are a small number of studies at present [7-11] and there is a lack of standardised reporting in serial MRI data across cohorts [12].

Following the standardisation work initiated with the Prostate Imaging Reporting and Data System (PI-RADS) guidelines, the Prostate Cancer Radiological Estimation of Change in Sequential Evaluation (PRECISE) panel was convened in 2016 [13]. The panel included ten experts in urology, eight in radiology and one in radiation oncology from the UK, Europe and North America and their objective was to define the conduct and reporting standards for patients on AS having serial MRI scans. A set of 394 statements relevant to prostate MRI reporting during AS was scored for agreement on a 9point scale by each panelist individually prior to the meeting. All scores were collated and consensus (or lack of consensus) for each statement was assessed using the RAND/UCLA criteria. Each statement was then discussed and rescored anonymously by each panelist during the face-to-face meeting, and the PRECISE checklist and case report template for reporting prostate MRI during AS were developed according to the consensus reached. The key features of the PRECISE recommendations are the measurement of each lesion at every time point, and a determination of the likelihood of radiological progression using a 1-to-5 Likert scale (PRECISE score) as shown in Table 1.

At present, there is limited literature on the application of the PRECISE recommendations in a clinical setting [14-17].

We report the application of the PRECISE recommendations in a large AS cohort of patients with serial MRI.

\section{Materials and methods}

At University College London Hospital, we have a cohort of $>620$ patients who have undertaken MRI-guided AS. The cohort was established in 2005 in a prospective manner and is defined by patients who have had a prostate MRI and a biopsy-confirmed low- to intermediate-risk PCa (i.e. Gleason $\leq 3+4$ and prostate-specific antigen (PSA) $\leq 20 \mathrm{ng} / \mathrm{ml}$ ) as per UK National Institute for Health and Care Excellence (NICE) guidelines [18], and who have chosen AS as their initial management option. No maximum cancer core length or number of positive cores was stipulated for 
Table 1 Assessment of likelihood of radiological progression on MRI in patients on active surveillance (PRECISE score)

\begin{tabular}{lll}
\hline PRECISE score & Assessment of likelihood of radiological progression & Example \\
\hline 1 & $\begin{array}{l}\text { Resolution of previous features suspicious on MRI } \\
\text { Reduction in volume and/or conspicuity of previous } \\
\text { features suspicious on MRI } \\
\text { Stable MRI appearance: no new focal/diffuse lesions }\end{array}$ & $\begin{array}{c}\text { Previously enhancing area no longer enhances } \\
\text { Reduction in size of previously seen lesion that remains } \\
\text { suspicious for clinically significant disease } \\
\text { Either no suspicious features or all lesions stable in size } \\
\text { and appearance }\end{array}$ \\
4 & $\begin{array}{c}\text { Significant increase in size and/or conspicuity of } \\
\text { features suspicious for prostate cancer } \\
\text { Definitive radiologic stage progression }\end{array}$ & $\begin{array}{c}\text { Lesion becomes visible on diffusion-weighted imaging; } \\
\text { significant increase in size of previously seen lesion }\end{array}$ \\
& Appearance of extracapsular extension, seminal vesicle \\
involvement, lymph node involvement, or bone metastasis
\end{tabular}

Legend - PRECISE: Prostate Cancer Radiological Estimation of Change in Sequential Evaluation; MRI: Magnetic Resonance Imaging. Reprinted from Moore CM, Giganti F, Albertsen P, et al (2017) Reporting magnetic resonance imaging in men on active surveillance for prostate cancer: the PRECISE Recommendations - a report of a European School of Oncology Task Force. European Urology, 71(4) 648-655. Copyright (2017), with permission from Elsevier (https://www.europeanurology.com)

eligibility, due in part to the extensive use of targeted biopsies that can result in 'risk inflation' and exclude patients from AS unnecessarily. We also note that the number of involved cores is not part of disqualification criteria from AS according to recent European and UK NICE guidelines [19, 20]. At our institution, all clinical records and MR images are routinely reviewed as part of an audit performed for the internal evaluation of the AS service (Fig. 1) and no institutional review board approval was required [21].

In detail, the timing of MRI on AS at our centre is based on both baseline risk (i.e. presence of a visible lesion at mpMRI) and PSA/PSA density changes during follow-up. Biopsy recommendations are based either on the suspicion of progression on MRI, or on adverse PSA kinetics without MRI changes. All biopsies are performed using a transperineal approach.

Data are updated to February 15, 2020.

\section{Primary outcome}

The primary outcome was to assess the relationship of the PRECISE score with clinical progression (defined by histological progression to $\geq$ GGG3 and/or initiation of active treatment).

\section{Secondary outcome}

The secondary outcome was to evaluate the relationship between PSA density and PRECISE score.

\section{MRI protocol}

All scans were performed according to international guidelines $[22,23]$. Three different scanners were used: two 1.5-T (Symphony or Avanto, Siemens) and one 3-T system (Achieva, Philips), with a pelvic phased-array coil. The protocol comprised T2-weighted, diffusion-weighted (including long $b$ sequences: $1400 \mathrm{~s} / \mathrm{mm}^{2}$ for $1.5-\mathrm{T}$ or $2000 \mathrm{~s} / \mathrm{mm}^{2}$ for 3$\mathrm{T}$ scanners) and dynamically contrast-enhanced imaging, as shown in Supplementary Table 1.

\section{MRI analysis and PRECISE score assessment}

For each patient, all scans were retrospectively re-reported by a dedicated expert radiologist (F.G.) with 7 years of experience in prostate cancer imaging (reporting $>1800$ prostate MRI scans/year) who had been actively involved in the writing of the PRECISE recommendations [13]. As per PRECISE recommendations, the radiologist was privy to PSA and initial biopsy results but blinded to the original MRI reports. The radiologist used a dedicated reporting tool (MIM®) Symphony Dx v. 6.8.3) that provides a customised workflow that leads the user to report according to the PRECISE recommendations using a step-by-step procedure, allowing the comparison of data from serial scans [24].

Each visible lesion was scored according to PI-RADS v. 2 guidelines at each time point [23]. At the second and subsequent scans, the radiologist assessed the PRECISE score for the likelihood of radiological progression from the last scan,

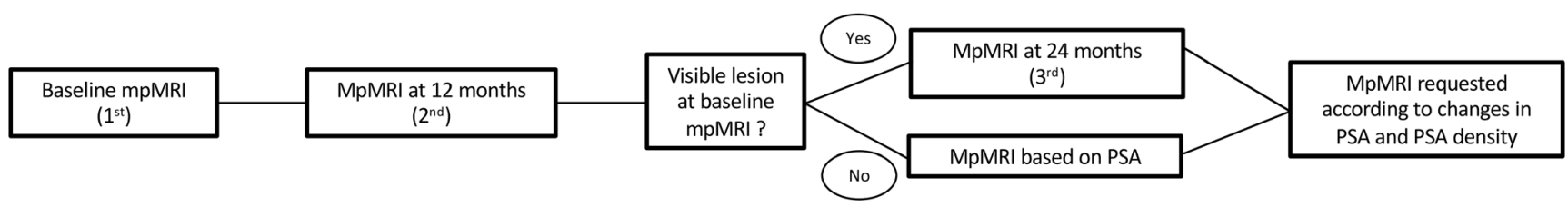

Fig. 1 Overview of the active surveillance MR protocol at University College London Hospital. Legend - MpMRI: multiparametric magnetic resonance imaging; PSA: prostate specific antigen 
using different MR features (conspicuity, increase in volume, signs of extracapsular extension or seminal vesicle invasion, etc.). A PRECISE score of 1 or 2 denotes radiological regression, a PRECISE 3 score indicates stability and a PRECISE 4 or 5 implies radiological progression (Table 1) [13].

As per PRECISE recommendations, the highest likelihood of clinically significant cancer of all separate lesions in a single scan provides the likelihood of clinically significant cancer for the whole prostate. Therefore, in the case of multifocal disease, the index lesion included in the analysis was the lesion with the highest PI-RADS score, and where there was more than one lesion at that score, the lesion with the highest volume was deemed the index lesion.

As there are still no explicit recommendations on which PRECISE score should be considered the most representative when multiple scans are acquired, we used the highest PRECISE score for each patient during the study period in this analysis. $[14,15]$

We applied the following interpretation to the PRECISE recommendations:

i) PRECISE 3: for those scans with a visible lesion showing stable MR features over time, or a persistent negative scan.

ii) PRECISE 4: for a new lesion in a previous negative scan or if a lesion was not visible at baseline MRI but appeared on a subsequent scan and then had stable MR features over time (i.e. in this case the highest PRECISE score was the most representative, as a new lesion developed).

\section{Statistical analysis}

Continuous variables were summarised by medians and interquartile ranges and the statistical significance of their differences was assessed using Mann-Whitney test. Categorical variables were summarised by frequencies and percentages. Wilcoxon test was applied to determine the statistical significance of differences.

The study asks whether there is an association between the PRECISE score and clinical progression. Because the PRECISE score could change over time with each successive scan at different time points, we included a time-dependent covariate for the PRECISE score using a Cox model predicting disease progression. Time zero was the date of the first biopsy showing PCa.

Progression-free survival was assessed using KaplanMeier curves and log-rank test was used to assess differences between curves.

The interaction term between each follow-up MRI and the corresponding PRECISE score, both considered timedependent covariates, was tested to explore the variation of
PSA density over time according to PRECISE score using linear regression and locally weighted scatterplot smoothing.

Statistical analyses were performed using $\mathrm{R}$ software (Version 3.4.2; Foundation for Statistical Computing).

All tests were two-sided, and $p<0.05$ was considered statistically significant.

\section{Results}

Our final cohort comprised a total of 553 patients on AS with two or more prostate MR scans, all of diagnostic quality (Fig. 2).

All scans $(n=2161)$ had been performed between December 2005 and January 2020.

Overall, 232/553 (42\%) patients were exclusively scanned on a $1.5-\mathrm{T}$ and $8 / 553(1.4 \%)$ exclusively on a 3 -T scanner. Supplementary Table 2 shows the number of patients and MR scans included in the study.

Table 2 shows baseline and follow-up characteristics of our population. Overall, 306/553 (55\%) patients had at least an additional biopsy, 178 (58\%) of which were targeted by visual registration. All follow-up biopsies were performed using a transperineal approach.

For patients with baseline PI-RADS 4 and 5 lesions $(n=$ $183), 133 / 183(73 \%)$ had Gleason $3+3$ and 50/183 (27\%) had Gleason $3+4$ at entry biopsy. Of them, 49/183 (27\%) had a targeted biopsy at entry.

\section{3 patients in the overall AS cohort}

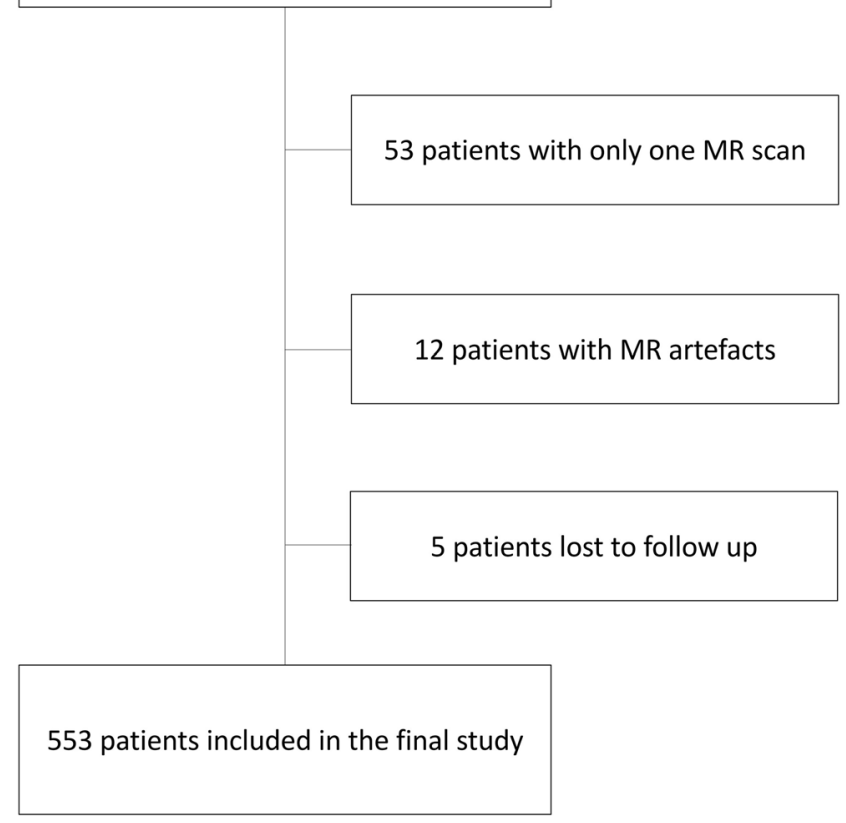

Fig. 2 Flowchart shows study enrolment. Legend - AS: active surveillance; MR: magnetic resonance 
Table 2 Descriptive statistics of all patients included in the study and stratified by biopsy progression, which is defined as one step in Gleason score upgrade (including those men with Gleason $3+3$ at entry and subsequent Gleason $3+4$ )

\begin{tabular}{|c|c|c|c|c|}
\hline & Overall $(n=553)$ & No biopsy progression $(n=177)$ & Biopsy progression $(n=129)$ & $p$ \\
\hline Age at diagnosis (years) & $62(56-67)$ & $62(57-66)$ & $62(56-67)$ & 0.60 \\
\hline PSA at baseline MR (ng/ml) & $6.3(4.7-8.4)$ & $6.51(4.88-8.30)$ & $6(4.7-8.6)$ & 0.47 \\
\hline Prostate volume at baseline MR (cc) & $45.8(32.7-63.4)$ & $47.93(36.15-64.38)$ & $38.32(29.3-56.7)$ & $<0.01$ \\
\hline PSA density at baseline MR (ng/ml/ml) & $0.12(0.09-0.2)$ & $0.12(0.09-0.17)$ & $0.15(0.1-0.21)$ & 0.01 \\
\hline $\begin{array}{l}\text { Gleason score at entry } \\
3+3\end{array}$ & $445[80]$ & $136[77]$ & 119 [92] & 0.02 \\
\hline $3+4$ & $108[20]$ & $41[23]^{*}$ & $10[8]$ & \\
\hline Biopsy type at entry & & & & - \\
\hline Transperineal template & $89[16]$ & $18[10]$ & 9 [7] & \\
\hline Transperineal + targeted & $76[14]$ & $11[6]$ & $13[10]$ & \\
\hline Systematic & $330[60]$ & $128[72]$ & $93[72]$ & \\
\hline Systematic + targeted & $35[6]$ & $12[7]$ & $8[6]$ & \\
\hline TURP & $23[4]$ & $8[5]$ & $6[5]$ & \\
\hline Baseline PI-RADS score & & & & $<0.01$ \\
\hline $1-2$ & $266[48]$ & $98[56]$ & $45[35]$ & \\
\hline 3 & 104 [19] & $36[20]$ & $23[18]$ & \\
\hline 4 & $164[30]$ & $36[20]$ & $54[42]$ & \\
\hline $5 * *$ & $19[3]$ & 7 [4] & $7[5]$ & \\
\hline Overall PRECISE score & & & & $<0.01$ \\
\hline 1 & $100[18]$ & $43[24]$ & $4[3]$ & \\
\hline 2 & $23[4]$ & $9[5]$ & $1[1]$ & \\
\hline 3 (non-visible lesion) & $152[28]$ & $50[28]$ & $5[4]$ & \\
\hline 3 (visible lesion) & $38[7]$ & $13[7]$ & $10[8]$ & \\
\hline 4 & $211[38]$ & $56[33]$ & 94 [73] & \\
\hline 5 & $29[5]$ & $6[3]$ & $15[11]$ & \\
\hline
\end{tabular}

Data are medians and interquartile range (parentheses); percentages in brackets [\%]. Legend - PSA: prostate-specific antigen; MR: magnetic resonance; TURP: transurethral ultrasound resection of the prostate; PI-RADS: Prostate Imaging Reporting and Data System; PRECISE: Prostate Cancer Radiological Estimation of Change in Sequential Evaluation

*18/41 [44\%] patients discontinued AS: 16/18 [89\%] patients showed radiological progression (fourteen PRECISE 4 and two PRECISE 5), fourteen of which showed also PSA progression. The remaining 2/18 [11\%] patients showed PSA progression but no radiological progression

**In the overall population, 13/19 [68\%] patients had Gleason $3+3$ and 6/19 [32\%] patients had Gleason $3+4$ at entry biopsy. In the 'no biopsy progression' group, $4 / 7$ [57\%] patients had Gleason $3+3$ and 3/7 [43\%] patients had Gleason $3+4$ at entry biopsy. In the 'biopsy progression' group, all patients $(7 / 7 ; 100 \%)$ had Gleason $3+3$ at entry biopsy. In terms of lesion location, $4 / 19$ [21\%] were anterior lesions (two left anterior, one right anterior, one midline anterior) and the other $15 / 19$ [79\%] lesions were in the peripheral zone ( 8 on the left and 7 on the right)

Table 3 lists the histopathological data of the whole population stratified by PRECISE score. If we have a closer look at those patients classified as PRECISE $4(n=211), 141 / 211$ $(67 \%)$ developed a new lesion, 36 of which $(26 \%)$ were upgraded on histology, whilst 70/211 (33\%) showed an increase in lesion size or conspicuity from baseline MRI, 58 of which (83\%) were upgraded on histology. More in detail, if we focus on those patients who showed biopsy progression in the PRECISE 4 group (94/211; 45\%), 36/94 (38\%) developed a new lesion whilst 58/94 (62\%) showed an increase in lesion size or conspicuity from baseline MRI. In this subcohort $(n=94)$, the median baseline PSA was $5.9 \mathrm{ng} / \mathrm{ml}(4.45-$ $8.55)$ and the median baseline PSA density was $0.15 \mathrm{ng} / \mathrm{ml} /$ $\mathrm{ml}(0.11-0.21)$.

\section{Primary outcome}

The association between PI-RADS score at baseline and the PRECISE score at follow-up scans is summarised in Table 4. In summary, PI-RADS 1-2 tended to be associated with PRECISE scores $\leq 3$ whilst patients with higher PI-RADS baseline scores (i.e. PI-RADS 4-5) had more than a twothirds chance of being attributed a PRECISE score 4 or 5 .

Median follow-up of the overall population was 76 months (52-100.5). In Fig. 3 a, we illustrate the proportion of patients that were free of clinical progression at 12, 24 and 60 months. Overall, 165/553 (30\%) patients experienced the primary outcome of clinical progression, with a median follow-up of those without clinical progression of 74.5 months (53-98), 


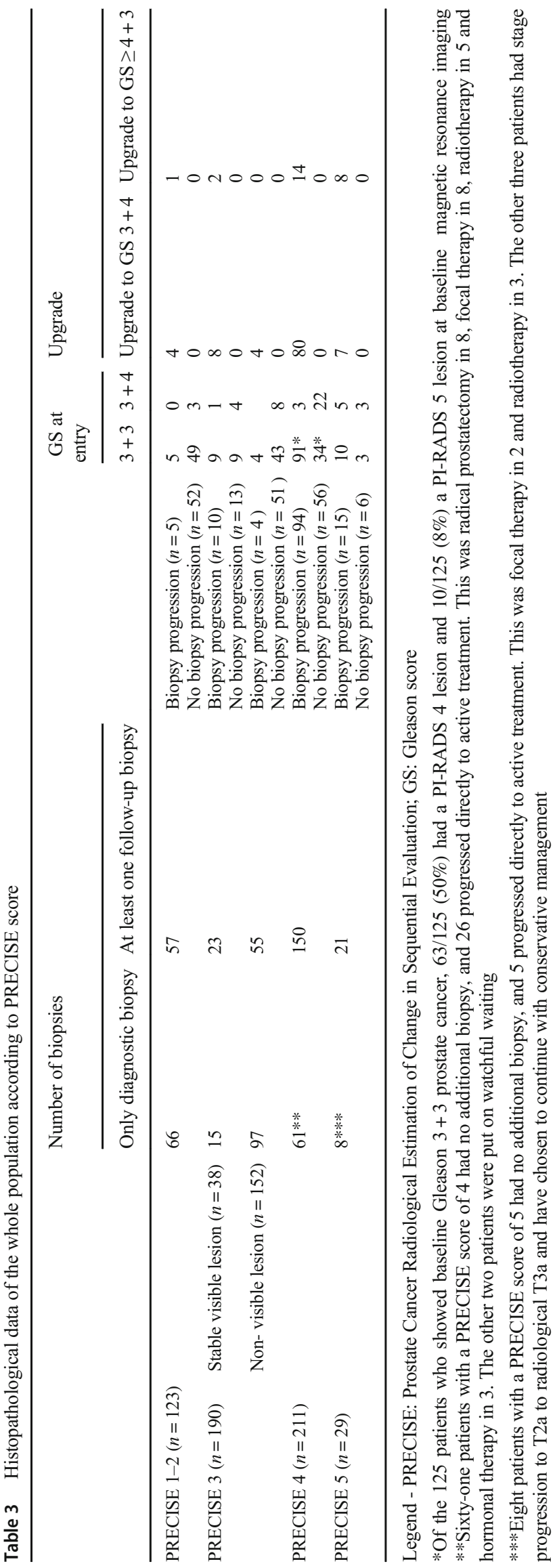

Table 4 Relationship between baseline PI-RADS score and the highest PRECISE score from scans for each patient in the overall population $(n=$ 553)

\begin{tabular}{lcccc}
\hline & PI-RADS 1-2 & PI-RADS 3 & PI-RADS 4-5 & Total \\
\hline PRECISE 1-2 & 34 & 55 & 34 & 123 \\
PRECISE 3 & 144 & 22 & 24 & 190 \\
PRECISE 4-5 & 88 & 27 & 125 & 240 \\
Total & 266 & 104 & 183 & 553 \\
\hline
\end{tabular}

Legend - PI-RADS: Prostate Imaging Reporting and Data System; PRECISE: Prostate Cancer Radiological Estimation of Change in Sequential Evaluation

as listed in Supplementary Table 3 and Supplementary Table 4.

For PRECISE 1-2, freedom from clinical progression was $100 \%$ at 12 and 24 months and $97 \%$ at 60 months. For PRECISE 3, freedom from clinical progression was $99 \%$ at 12 and 24 months and $97 \%$ at 60 months and for PRECISE 4 5 it was $96 \%, 91 \%$ and $61 \%$, respectively. There was a significant difference in clinical progression between PRECISE $1-2$ vs $4-5$ and PRECISE 3 vs $4-5(p<0.001)$.

The proportion of patients free from histological progression to $\geq$ GGG 3 or initiation of active treatment (analysed separately) is shown in Fig. 3 b and c, respectively.

The Cox model predicting disease progression with a timedependent covariate for the PRECISE score is shown in Supplementary Table 5. Supplementary Table 6 shows the number of patients with biopsy progression stratified according to a negative MR scan or radiological progression before biopsy.

\section{Secondary outcome}

There was a relationship between change in PSA density and the PRECISE score. The interaction term between the PRECISE score and each follow-up MRI in predicting PSA density variation over time was significant for PRECISE 4-5 (hazard ratio 1.04; $p=0.01$ ) (Fig. 4 and Supplementary Table 7).

Table 5 reports the quantitative and qualitative parameters used to assess the PRECISE score for each patient included in the study in accordance with the PRECISE case report form.

Figure 5 shows a case classified as PRECISE 4.

\section{Discussion}

This is the first report that applies the PRECISE criteria for radiological progression in a UK cohort of patients with $\mathrm{PCa}$ on an MRI-led AS programme.

We have shown that radiological stability is associated with 97\% freedom from clinical progression at 5 years. We have also demonstrated that a PI-RADS score of 4 or 5 at entry to 


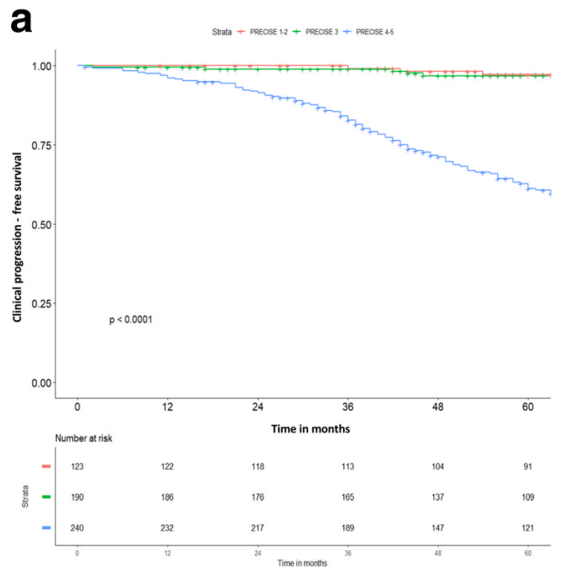

Fig. 3 Kaplan-Meier curves showing the rate of clinical progression $(\geq$ Gleason Grade Group 3 and initiation of active treatment) (a), only biopsy progression ( $\geq$ Gleason Grade Group 3) (b) and only initiation of active

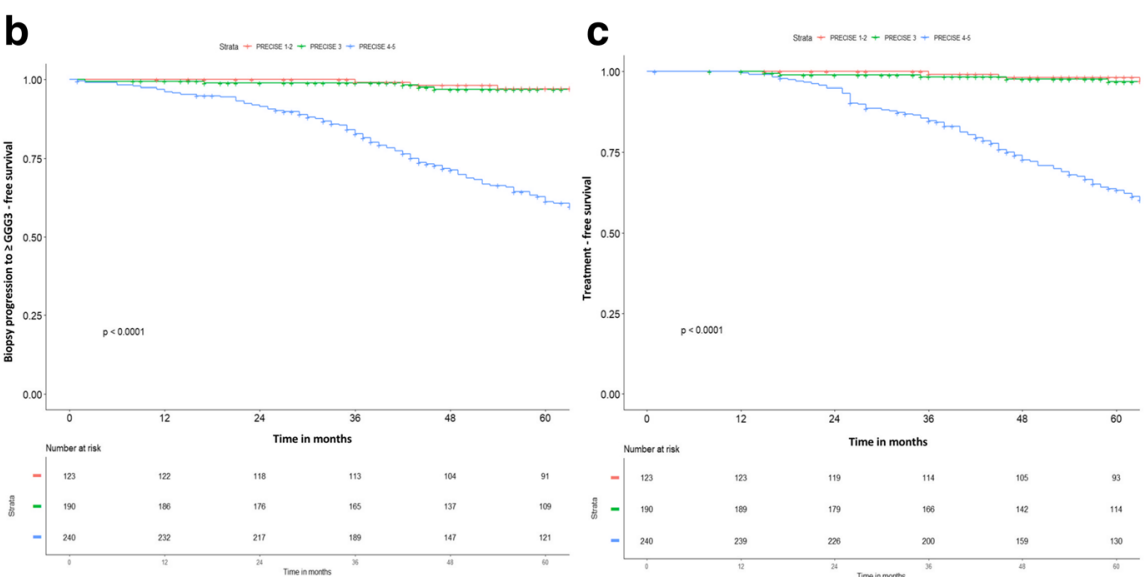

treatment (c) stratified by PRECISE score (1-2 vs 3 vs $4-5)$ in the overall population. Legend - GGG: Gleason Grade Group

treatment. As they had already met the criteria for choosing active treatment at baseline, this approach was permitted.

We therefore chose a combined endpoint of either histological progression to GGG 3, or initiation of active treatment as the definition of clinical progression.

In our MRI-led AS programme, biopsies are not offered on a protocol basis. Instead they are offered to patients 'for cause'. The triggers for the recommendation comprise adverse changes in MRI or PSA kinetics. This approach was developed in response to our work in this field, where we demonstrated a high negative predictive value of MRI for GGG 3 disease. Whilst this protocol differs from all other published protocols, we know that in practice, compliance rates with protocol biopsy can be as low as 20 to $30 \%$, in both routine practice and formal studies such as PRIAS [25], and actual
Fig. 4 Multivariable relationship between each follow-up MRI and PSA density over time stratified by PRECISE score (1-2 vs 3 vs 4-5) in the overall population. Legend - PSA, prostate specific antigen; FU: follow-up

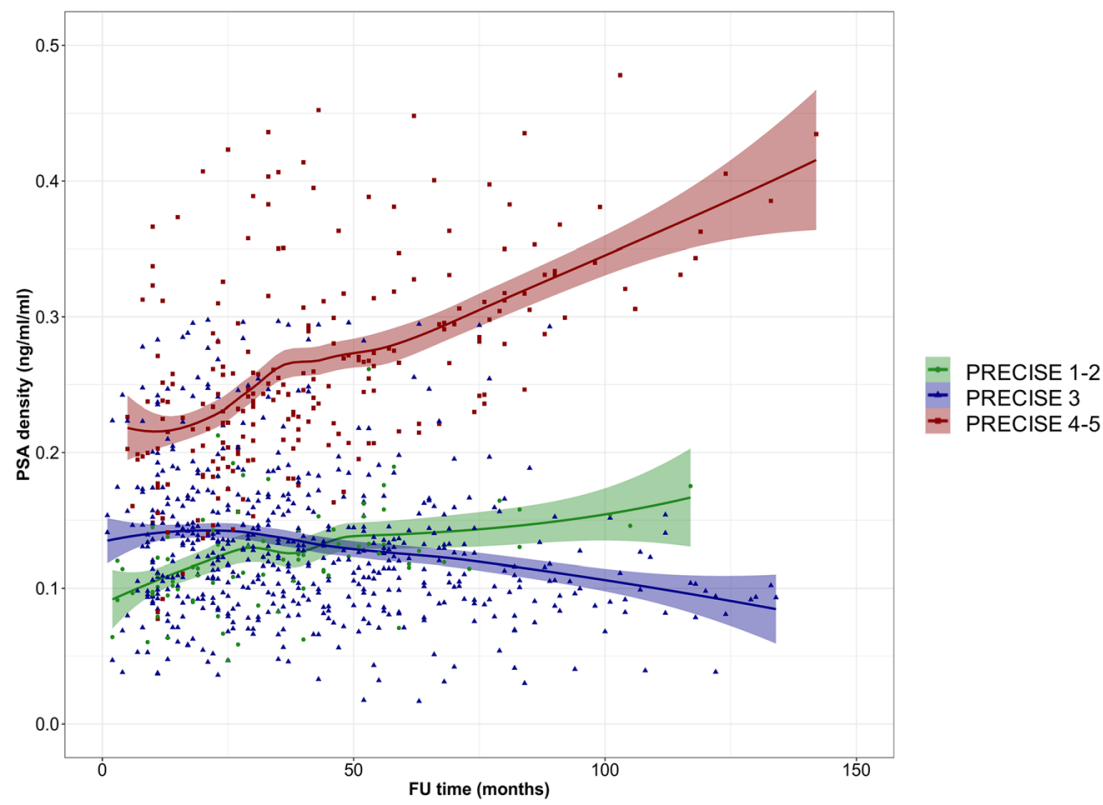




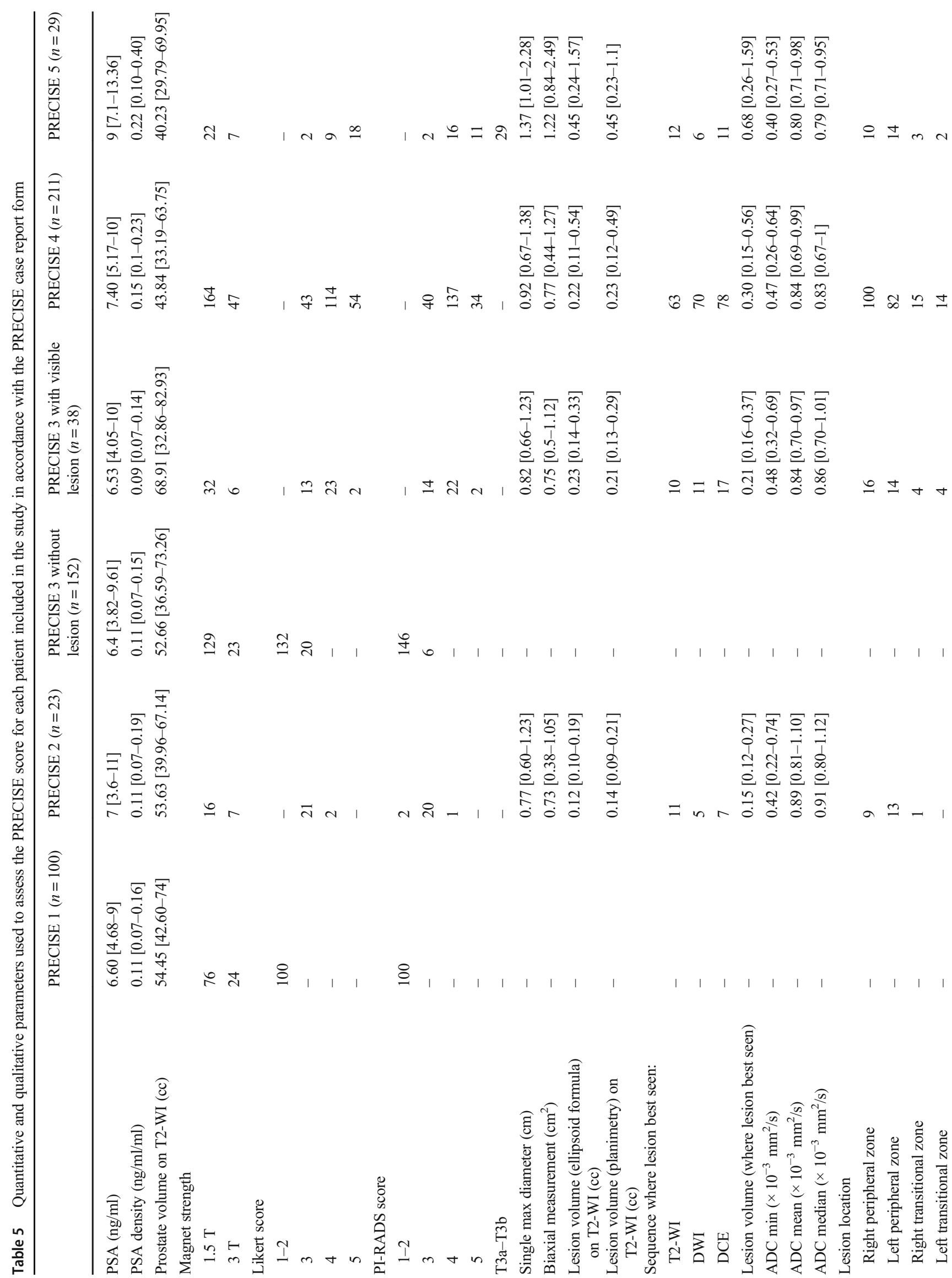




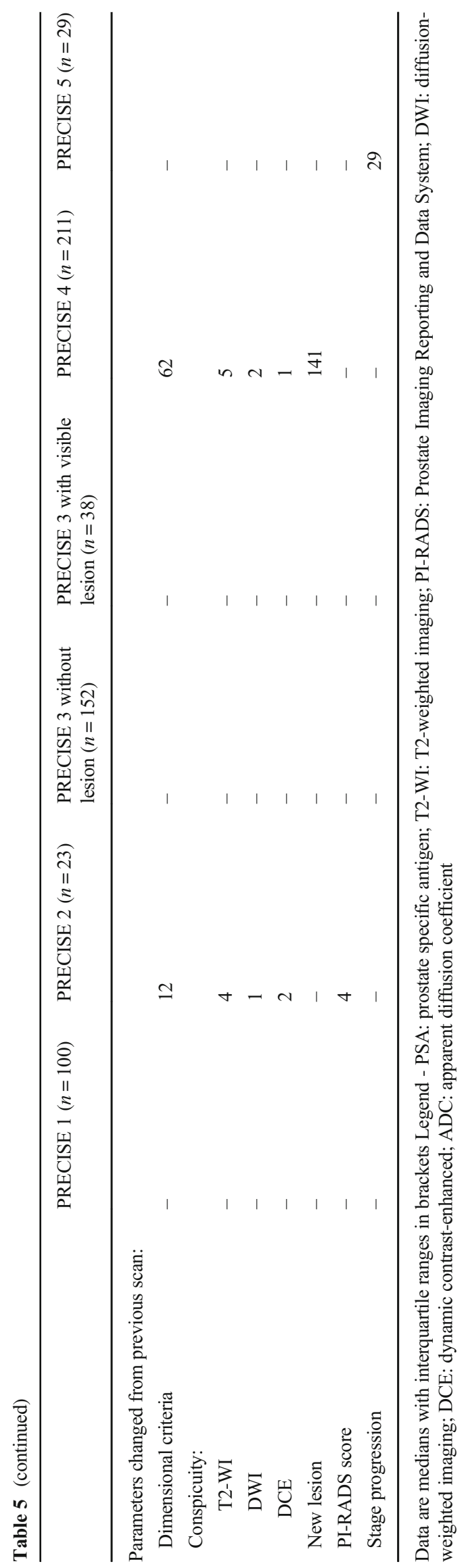

biopsy rates may be more similar in the two approaches than the protocols would suggest $[26,27]$.

Whilst some would see the lack of additional biopsy as a drawback, we believe that it has significant advantages in terms of patient acceptability and compliance, and that the risk of 'loss of opportunity for cure' is no higher using this approach than standard biopsy alone. For example, in our cohort, $1 / 553$ patients $(<1 \%)$ developed nodal disease and 2/553 $(<1 \%)$ patients had bone metastases whilst on surveillance, with median follow-up of 6.3 years. This compares well to the Sunnybrook cohort [28], where 13/980 (1.3\%) patients developed lymph node disease, and 18/980 (1.8\%) patients developed bone metastases, with a median follow-up of 6.3 years.

\section{Limitations}

The first, and possibly the most relevant, limitation is that the entry biopsy was often TRUS-guided and without a clear definition of the lesion location, whilst subsequent biopsies were done using a transperineal approach and this could represent a possible bias. Since this is an image-guided AS cohort, not all patients underwent re-biopsy during follow-up and resampling was often triggered by apparent tumour growth on mpMRI. As this is a retrospective study, this means that patients with negative mpMRI results did not routinely undergo biopsies and we acknowledge that this factor could contribute to verification bias [29].

However, we know that the likelihood of clinically significant PCa with negative MRI is low and such patients are unlikely to benefit from a biopsy [30].

We should also mention that patients entering AS at our centre with a diagnostic biopsy at another centre are required to have a concordant mpMRI and biopsy and undergo repeat biopsy to assess any discordance if required.

We acknowledge that this is a single-centre study in a centre with significant prostate mpMRI experience, and the results may not be generalisable to all centres.

Only a single radiologist, although highly experienced in prostate MR reporting, applied the PRECISE score in the whole cohort. However, we have recently demonstrated the high interobserver agreement of the PRECISE score between two expert radiologists in a multicentre study [14].

The retrospective assessment of the PRECISE score could be seen as another limitation, as we have been using the PRECISE score only from 2016 onwards but the assessment of radiological progression before that date was subjective and not well-standardised. However, after completion of the study, we observed that $>95 \%$ of the original reports were in line with the PRECISE scores that were retrospectively assessed, corroborating our current results.

Lastly, MRI quality has improved over time, and the earliest scans might be less informative than the most recent ones. Some patients received scans on both 1.5-T and 3-T scanners, 


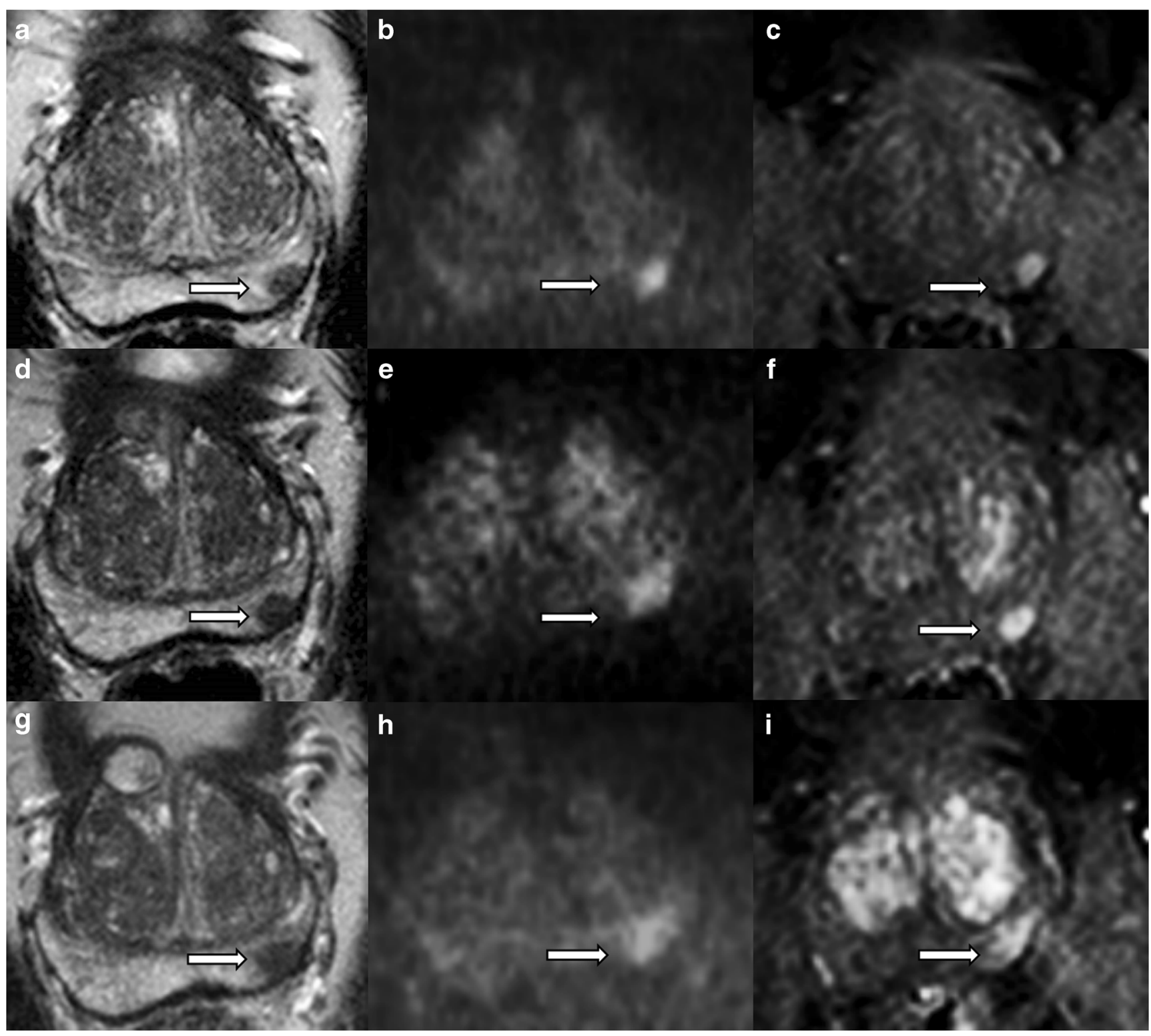

Fig. 5 Fifty-year-old man on active surveillance for Gleason $3+3$ $(4 \mathrm{~mm})$ prostate cancer in the left peripheral zone on standard transrectal ultrasound biopsy. The mpMRI scan at baseline $(\mathbf{a}, \mathbf{b}, \mathbf{c})$ shows a leftsided peripheral zone lesion (arrows) between 4 and 5 o'clock characterised by low signal intensity on T2-weighted imaging (a),

and this may have limited the ability to accurately compare measurements of lesions between scans.

\section{Implications for AS programmes}

AS programmes typically have more intense follow-up in the early period, to identify patients who were misclassified. There has long been controversy over the use of PSA kinetics in patients on AS, with some reports suggesting that PSA kinetics are not predictive for biopsy upgrade [31].

In this cohort, we demonstrate an association of change in PSA density with radiological progression. If these findings restricted diffusion on diffusion-weighted imaging (b) and early enhancement on dynamic contrast-enhanced imaging (c). The lesion showed progressive radiological change in volume and conspicuity after one $(\mathbf{d}, \mathbf{e}, \mathbf{f})$ and two $(\mathbf{g}, \mathbf{h}, \mathbf{i})$ years (PRECISE score: 4$)$. The patient was treated with high-intensity focused ultrasound

are confirmed in larger cohorts, we may be able to use PSA density as a trigger for further investigation rather than a protocol-based approach using time from diagnosis.

\section{Conclusions}

Our study suggests that the widespread use of the PRECISE recommendations might have two main clinical consequences: (i) identification of patients on AS who progress (PRECISE 4-5) in a timely manner, promoting re-biopsy/ treatment; (ii) avoidance of repeat biopsy (and a lower 
intensity surveillance schedule) for patients with PRECISE 13 , reducing the burden of surveillance for the individual and the healthcare system.

We do not promote the adoption of our AS protocol in every clinical setting, but we provide here the first application of the PRECISE recommendations in a cohort of patients monitored according to the UK NICE guidelines.

Future randomised studies (e.g. a study design of 'biopsy' versus 'no biopsy' according to PRECISE, with presence or absence of clinical progression as the ultimate reference) will be more informative.

We acknowledge that the PRECISE score will require further validation in prospective multicentre cohorts, using a range of reporters. Further analysis in larger cohorts, including the Movember GAP3 cohort of over 15,000 patients on AS across 25 countries, is planned [2].

Funding This work has been funded by the UCL Graduate Research Scholarship and the Brahm PhD scholarship in memory of Chris Adams.

\section{Compliance with ethical standards}

Guarantor The scientific guarantor of this publication is Dr. Francesco Giganti.

Conflict of interest The authors of this manuscript declare relationships with the following companies:

Francesco Giganti is funded by the UCL Graduate Research Scholarship and the Brahm PhD scholarship in memory of Chris Adams.

Vasilis Stavrinides is supported by an MRC Clinical Research Training Fellowship (MR/S005897/1) and has been previously supported by a UCL Bogue Fellowship and an EACR Travel Fellowship.

Bruce Trock has the following disclosures: Myriad Genetics, Inc.research grant funding, advisory board; MDxHealth, Inc. - research grant funding; GenomeDX Biosciences, Inc.- consulting.

Shonit Punwani receives research support from the United Kingdom's National Institute of Health Research (NIHR) UCLH/UCL Biomedical Research Centre.

Alex Kirkham is supported by the UCLH/UCL Biomedical Research Centre.

Mark Emberton is a United Kingdom National Institute of Health Research (NIHR) Senior Investigator and receives research support from the UCLH/UCL NIHR Biomedical Research Centre.

Caroline M Moore is supported by the UK NIHR, Movember, PCUK and the EAU Research Foundation.

Statistics and biometry Three of the authors (AS, VS and BJT) has significant statistical expertise.

Informed consent Written informed consent was not required for this study because at our institution all clinical records and MR images are routinely reviewed as part of an audit performed for the internal evaluation of the AS service as per UK NICE guidelines.

Ethical approval Institutional Review Board approval was not required because at our institution all clinical records and MR images are routinely reviewed as part of an audit performed for the internal evaluation of the AS service as per UK NICE guidelines.
Study subjects or cohorts overlap Some study subjects or cohorts have been previously reported in:

- Giganti F, et al Prostate Cancer Prostatic Dis (2018); 21(4):556-563.

- Giganti F, et al Eur Radiol (2020); 30(4):2082-2090.

- Giganti F, et al Magn Res Imag (2020); 67:50-58.

\section{Methodology}

- retrospective

- diagnostic or prognostic study

- performed at one institution

Open Access This article is licensed under a Creative Commons Attribution 4.0 International License, which permits use, sharing, adaptation, distribution and reproduction in any medium or format, as long as you give appropriate credit to the original author(s) and the source, provide a link to the Creative Commons licence, and indicate if changes were made. The images or other third party material in this article are included in the article's Creative Commons licence, unless indicated otherwise in a credit line to the material. If material is not included in the article's Creative Commons licence and your intended use is not permitted by statutory regulation or exceeds the permitted use, you will need to obtain permission directly from the copyright holder. To view a copy of this licence, visit http://creativecommons.org/licenses/by/4.0/.

\section{References}

1. Bruinsma SM, Bangma CH, Carroll PR et al (2016) Active surveillance for prostate cancer: a narrative review of clinical guidelines. Nat Rev Urol 13(3):151-167

2. Bruinsma SM, Zhang L, Roobol MJ et al (2018) The Movember Foundation's GAP3 cohort: a profile of the largest global prostate cancer active surveillance database to date. BJU Int 121(5):737-744

3. Giganti F, Moore CM (2018) Magnetic resonance imaging in active surveillance - a modern approach. Transl Androl Urol 7(1):116131

4. Schoots IG, Nieboer D, Giganti F, Moore CM, Bangma CH, Roobol MJ (2018) Is magnetic resonance imaging-targeted biopsy a useful addition to systematic confirmatory biopsy in men on active surveillance for low-risk prostate cancer? A systematic review and meta-analysis. BJU Int 122(6):946-958

5. Klotz L, Loblaw A, Sugar L et al (2018) Active Surveillance Magnetic Resonance Imaging Study (ASIST): results of a randomized multicenter prospective trial. Eur Urol 75(2):300-309

6. Klotz L, Pond G, Loblaw A et al (2020) Randomized study of systematic biopsy versus magnetic resonance imaging and targeted and systematic biopsy in men on active surveillance (ASIST): 2year postbiopsy follow-up. Eur Urol 77(3):311-317

7. Felker ER, Wu J, Natarajan S et al (2016) Serial magnetic resonance imaging in active surveillance of prostate cancer: incremental value. J Urol 195(5):1421-1427

8. Giganti F, Moore CM, Punwani S, Allen C, Emberton M, Kirkham A (2018) The natural history of prostate cancer on MRI: lessons from an active surveillance cohort. Prostate Cancer Prostatic Dis 21(4):556-563

9. Tamada T, Dani H, Taneja SS, Rosenkrantz AB (2017) The role of whole-lesion apparent diffusion coefficient analysis for predicting outcomes of prostate cancer patients on active surveillance. Abdom Radiol (NY) 42(9):2340-2345

10. Moore CM, Robertson NL, Jichi F et al (2017) The effect of dutasteride on magnetic resonance imaging defined prostate cancer: MAPPED - a randomized, placebo controlled, double-blind clinical trial. J Urol 197(4):1006-1013 
11. Giganti F, Moore CM, Robertson NL et al (2017) MRI findings in men on active surveillance for prostate cancer: does dutasteride make MRI visible lesions less conspicuous? Results from a placebo-controlled, randomised clinical trial. Eur Radiol 27(11):47674774

12. Schoots IG, Petrides N, Giganti F et al (2015) Magnetic resonance imaging in active surveillance of prostate cancer: a systematic review. Eur Urol 67(4):627-636

13. Moore CM, Giganti F, Albertsen P et al (2017) Reporting magnetic resonance imaging in men on active surveillance for prostate cancer: the PRECISE recommendations - a report of a European School of Oncology Task Force. Eur Urol 71(4):648-655

14. Giganti F, Pecoraro M, Stavrinides V et al (2020) Interobserver reproducibility of the PRECISE scoring system for prostate MRI on active surveillance: results from a two-centre pilot study. Eur Radiol 30(4):2082-2090

15. Giganti F, Pecoraro M, Fierro D et al (2020) DWI and PRECISE criteria in men on active surveillance for prostate cancer: a multicentre preliminary experience of different ADC calculations. Magn Reson Imaging 67:50-58

16. Dieffenbacher S, Nyarangi-Dix J, Giganti F et al (2019) Standardized magnetic resonance imaging reporting using the prostate cancer radiological estimation of change in sequential evaluation criteria and magnetic resonance imaging/transrectal ultrasound fusion with transperineal saturation biopsy to select men. Eur Urol Focus S2405-4569(19)30076-8 (in press). https://doi.org/10. 1016/j.euf.2019.03.001

17. Ullrich T, Arsov C, Quentin M et al (2020) Multiparametric magnetic resonance imaging can exclude prostate cancer progression in patients on active surveillance: a retrospective cohort study. Eur Radiol 30(4):2082-2090

18. NICE. Prostate cancer diagnosis and management: Clinical Guideline 175. 2014;(January). Accessed on 27 Mar 2020. https://www.nice.org.uk

19. Robertson NL, Hu Y, Ahmed HU, Freeman A, Barratt D, Emberton M (2014) Prostate cancer risk inflation as a consequence of imagetargeted biopsy of the prostate: a computer simulation study. Eur Urol 65(3):628-634

20. EAU Guidelines. Edn. presented at the EAU Annual Congress Amsterdam 2020. ISBN 978-94-92671-07-3

21. Stavrinides V, Giganti F, Trock B et al (2020) Five-year outcomes of magnetic resonance imaging-based active surveillance for prostate cancer: a large cohort study. Eur Urol 78(3):443-451
22. Barentsz JO, Richenberg J, Clements R et al (2012) ESUR prostate MR guidelines 2012. Eur Radiol 22(4):746-757

23. Weinreb JC, Barentsz JO, Choyke PL et al (2016) PI-RADS prostate imaging - reporting and data system: 2015, version 2. Eur Urol 69(1): $16-40$

24. Giganti F, Allen C, Piper JW et al (2018) Sequential prostate MRI reporting in men on active surveillance: initial experience of a dedicated PRECISE software program. Magn Reson Imaging 57:34 39

25. Bokhorst LP, Valdagni R, Rannikko A et al (2016) A decade of active surveillance in the PRIAS study: an update and evaluation of the criteria used to recommend a switch to active treatment. Eur Urol 70(6):954-960

26. Loeb S, Walter D, Curnyn C, Gold HT, Lepor H, Makarov DV (2016) How active is active surveillance? Intensity of followup during active surveillance for prostate cancer in the United States. J Urol 196(3):721-726

27. Evans MA, Millar JL, Earnest A et al (2018) Active surveillance of men with low risk prostate cancer: evidence from the Prostate Cancer Outcomes Registry-Victoria. Med J Aust 208(10):439-443

28. Yamamoto T, Musunuru B, Vesprini D et al (2016) Metastatic prostate cancer in men initially treated with active surveillance. $\mathrm{J}$ Urol 195(5):1409-1414

29. Tangen CM, Goodman PJ, Till C, Schenk JM, Lucia MS, Thompson IM Jr (2016) Biases in recommendations for and acceptance of prostate biopsy significantly affect assessment of prostate cancer risk factors : results from two large randomized clinical trials. J Clin Oncol 34(36):4338-4344

30. Moldovan PC, Van Den Broeck T, Sylvester R et al (2017) What is the negative predictive value of multiparametric magnetic resonance imaging in excluding prostate cancer at biopsy ? A systematic review and meta-analysis from the European Association of Urology Prostate Cancer Guidelines Panel. Eur Urol 72:250-266

31. Ross AE, Loeb S, Landis P et al (2010) Prostate-specific antigen kinetics during follow-up are an unreliable trigger for intervention in a prostate cancer surveillance program. J Clin Oncol 28(17): 2810-2816

Publisher's note Springer Nature remains neutral with regard to jurisdictional claims in published maps and institutional affiliations. 